\title{
Antifungal screening of 61 folkloric medicinal plant extracts against dermatophytic fungi Tridhophyton rubrum
}

\author{
P. Shivakumar Singh, G.M. Vidyasagar*
}

Medicinal Plants and Microbiology Research Laboratory, Department of Post Graduate Studies and Research in Botany, Gulbarga University, Gulbarga 585 106, Karnataka, India.

\section{ARTICLE INFO \\ Article history: \\ Received on: 04/02/2015 \\ Revised on: 22/02/2015 \\ Accepted on: 18/03/2015 \\ Available online: 27/05/2015}

Key words:

Folkloric medicinal plants,

Trichophyton rubrum,

antifungal screening.

\begin{abstract}
A series 305 extracts from 61 traditional medicinal plants (belonging to 33 different families) used in treating skin diseases in Hyderabad Karnataka region were subjected to antidermatophytic screening against Trichophyton rubrum (MTCC 1344). Pet ether, chloroform, ethyl acetate, methanol and aqueous extracts of each plant were tested for their antifungal activity using agar well diffusion method at sample concentration of 5 \& 2.5 $\mathrm{mg} / \mathrm{ml}$. The results indicated that out of 61 plants, 18 exhibited very effective antidermatophytic activity in methanolic extracts, effective activity observed in 13 plants in different extracts, whereas 26 plants showed moderate activity, 04 plants showed weak activity. The minimum inhibitory concentrations of 18 very effective plants were determined. On the basis of the results obtained, the crude extracts of Allium sativam Linn., Annona reticulata L., Annona squamosa L., Argemone mexicana L., Butea monosperma, Ceasalpinia bonducella, Citrus medica L., Corchorus oleterius L., Emblica officinalis, Euphorbia tirucalli L., Ficus racemosa L., Gymnosporia montana, Lawsonia inermis L., Solanum nigrum L., Sterculia foetida L., Tribulus terrestris L., Vitex negundo L., and Zingiber officinale exhibited significant antidermatophytic activity (T. rubrum) and properties that support folkloric use in the treatment of skin diseases as broad-spectrum antimycotic agents. This probably explains the use of these plants by the indigenous people against dermatological infections.
\end{abstract}

\section{INTRODUCTION}

Medicinal plants represent a rich source of antimicrobial agents (Mahesh \& Satish, 2008). Many of the plant materials used in traditional medicine are readily available in rural areas at relatively cheaper than modern medicine (Mann et al., 2008). Plants generally produce many secondary metabolites which constitute an important source of microbicides, pesticides and many pharmaceutical drugs. Plant products still remain the principal source of pharmaceutical agents used in traditional medicine (Ibrahim, 1997; Ogundipe et al., 1998). This is because of a broader degree of chemical diversity and novelty of molecules found in natural products than that from another source. Fungal diseases have historically been a difficult clinical entity to effectively deal with. The available drugs to treat fungal infections have been limited. Furthermore, in this

\footnotetext{
* Corresponding Author

VIDYASAGAR GM, Medicinal Plants and Microbiology Research Laboratory, Department of Post Graduate Studies and Research in Botany, Gulbarga University, Gulbarga - 585 106, Karnataka, India. Email: gm.vidyasagar@rediffmail.com
}

armamentarium there are problems with side effects, the rapid development of resistance, drug interactions and fungistatic mechanism of action. Thus, there is a need for the development of more efficacious antidermatophytic agents. Plants are the best source for identification of leads for the development of novel drug compounds (Raginee Verma, Satsangi G, 2011).

The antifungal activity of Indian medicinal plants has not been extensively studied and was described in only a few reports (Sasidharan, 1997). The dermatophytes are a group of closely related fungi that have the capacity to invade keratinized tissue (skin, hair and nails) of humans and other animals to produce an infection, dermatophytosis, commonly referred to as ringworm. They are classified in three anamorphic genera, Epidermophyton, Microsporum and Trichophyton.

The vast majority of chronic dermatophyte infection of human skin are caused by Trichophyton rubrum (Weitzman I and Summerbell RC 1995). Trichophyton rubrum infect hairs, skins and nails (David et al., 1997). Human beings are the main or only host for anthropophilic dermatophytes like T. rubrum. (Ananthanarayan and Paniker. 2009, Sumit Kumar, Shrikara Mallya P, 2012, Ogu G. I., etal., 2011) 
The past three decades have seen a dramatic increase in microbial resistance to antimicrobial agents (Chopra et al., 1996; Baquero, 1997).

There were no previous reports using folklore medicinal plants against $T$. rubrum from this region. Therefore, in the present report the antibiograme against a universal dermatophytic fungi $T$. rubrum response reported.

\section{MATERIALS AND METHODS}

\section{Plant materials}

Plant materials were collected from various localities of Hyderabad Karnataka region and Identified with the help of Gulbarga district flora (Seetharam et al., 2000) the voucher specimens deposited in herbarium centre, Department of Botany, Gulbarga University, Karnataka, India. The collected plant materials were initially rinsed with distilled water to remove soil and other contaminants and dried on paper towel in the laboratory at $37 \pm 2^{\circ} \mathrm{C}$ for a week.

\section{Preparation of the plant extracts}

The selected plant part materials after shade drying were ground in a grinding machine in the laboratory. $25 \mathrm{~g}$ of shade dried powder was weighed and extracted successively with petroleum ether, chloroform, ethyl acetate, methanol and aqueous in soxhlet extractor for $48 \mathrm{~h}$. The extracts were concentrated under reduced pressure and preserved in refrigerator in airtight bottles for further use.

\section{Microbial culture and growth conditions}

Trichophyton rubrum (MTCC 1344) was used as test microorganism. Culture of T. rubrum grown on Sabouraud dextrose broth (HiMedia) at $28{ }^{\circ} \mathrm{C}$ for $48 \mathrm{~h}$ and it was maintained on agar slants at $4^{\circ} \mathrm{C}$.

\section{Inoculum preparation}

Stock inoculums of T. rubrum was prepared from 10-day cultures in PDA at $28{ }^{\circ} \mathrm{C}$ to induce sporulation. Fungal colonies were covered with $5 \mathrm{ml}$ of sterile saline solution $(\mathrm{NaCl} 0.85 \%$ w/v), the surface gently scraped with a sterile loop and the resultant mixture of fungal units was then transferred to a sterile tube.

The turbidity of the final inoculum was standardized according to a McFarland scale 0.5 tube and adjusted to a fungal population of 106 colony former units (CFU). The confirmation of inoculum quantification was done by plating $0.01 \mathrm{ml}$ of inoculum suspension in Sabouraud dextrose agar (SDA). The dishes were incubated at $28{ }^{\circ} \mathrm{C}$ and examined daily for the presence of fungal colonies which were counted as soon as growth became visible (Santos et al., 2006; Hadacek, Greger, 2000).

\section{Agar-well diffusion method}

The assay was conducted by agar well diffusion method. About 15 to $20 \mathrm{ml}$ of potato dextrose agar medium was poured in the sterilized petridishes and allowed to solidify. Fungal lawn was prepared using 5 days old culture strains. The fungal strains were suspended in a saline solution $(0.85 \% \mathrm{NaCl})$ and adjusted to a turbidity of $0.5 \mathrm{Mac}$ Farland standards (108 CFU/ml). $1 \mathrm{ml}$ of fungal strain was spread over the medium using a sterilized glass spreader. Using flamed sterile borer, wells of $4 \mathrm{~mm}$ diameter were punctured in the culture medium and required concentrations of serially diluted extract $(2.5,5 \mathrm{mg} / \mathrm{ml})$ was added to the $20 \mu \mathrm{l}$ to each wells.

The plates thus prepared were left for diffusion of extracts into media for one hour in the refrigerator and then incubated at $30^{\circ} \mathrm{C}$. After incubation for $48 \mathrm{~h}$, the plates were observed for zone of inhibition. Diameter zone of inhibition was measured and expressed in millimeters. Dimethyl formamide (DMF) was used as a negative control. The experiments were conducted in triplicates (Magaldi, 2004)./

\section{Minimum Inhibitory Concentration}

One $\mathrm{ml}$ of sterile liquid Sabouraud medium was added to 08 sterile capped tubes, $1 \mathrm{ml}$ of each solvent extracts suspension was added to tube 1 . The contents were mixed and $1 \mathrm{ml}$ was transferred to tube 2 .

This serial dilution was repeated through to tube six and $1 \mathrm{ml}$ was discarded from tube 6 . Fifty $\mu \mathrm{l}$ of inoculum was added to tubes 1-8 and the contents were mixed. Medium control (no inoculum and no drug) and inoculum control (no drug) tubes were prepared. The final concentrations of each plant, solvent extract ranged from $05 \mathrm{mg} / \mathrm{ml}$ to $0.15 \mathrm{mg} / \mathrm{ml}$.

The tubes were incubated at $30^{\circ} \mathrm{C}$ for $96 \mathrm{~h}$. The fungal growth in each tube was evaluated visually depending upon the turbidity in the tubes. MIC was defined as the drug concentration at which the turbidity of the medium was the same as the medium control. (NCCLS-1997)

\section{Preliminary screening tests for secondary metabolites:}

Preliminary tests, for the detection of secondary metabolites, were carried out for 305 extracts of 61 plants by adopting standard methods (Harborne, 1998).

\section{Statistical Analysis}

All the experiments were conducted in triplicate unless stated otherwise and statistical analysis of the data was performed by analysis of variance (ANOVA), using STATISTICA 5.5 (Stat Soft Inc, Tulsa, Oklahoma, USA) software. A probability value of difference $\mathrm{p} \sim 0.05$ was considered to denote a statistically significant all data were presented as mean values \pm standard deviation (SD).

\section{RESULTS AND DISCUSSION}

The plant extracts and their level of activity against Trichophyton rubrum was listed in Table 1. A series of 305 extracts from 61 ethno medicinal plants (parts were shown in figure. 1) Belonging to 33 different families were used in treating 
Table 1: Antifungal screening of 61 medicinal plant extracts against a common dermatophytic fungi Trichophyton rubrum.

\begin{tabular}{|c|c|c|c|c|c|c|c|c|c|c|c|c|c|c|}
\hline \multirow{3}{*}{$\begin{array}{l}\text { Sl. } \\
\text { No. }\end{array}$} & \multirow{3}{*}{ Name of the Plant } & \multirow{3}{*}{ PU } & \multicolumn{10}{|c|}{ Zone of Inhibition in different solvents (mm) } & \multirow{3}{*}{$\begin{array}{l}\text { Control } \\
\text { DMSO } \\
\end{array}$} & \multirow{3}{*}{$\begin{array}{l}\text { Standard } \\
\text { Ketoconazole }\end{array}$} \\
\hline & & & \multicolumn{2}{|c|}{ Petroleum Ether } & \multicolumn{2}{|l|}{ Chloroform } & \multicolumn{2}{|l|}{ Ethyl estate } & \multirow{2}{*}{$\frac{\text { Methanol }}{\text { A }}$} & & Aqueous & & & \\
\hline & & & $\mathbf{A}$ & $\mathbf{B}$ & $\mathbf{A}$ & B & $\mathbf{A}$ & B & & B & $\mathbf{A}$ & $\mathbf{B}$ & & \\
\hline$\overline{01}$ & Achyranthes aspera $\mathrm{L}$ & $\mathrm{L}$ & $06.66 \pm 1.15$ & $05.33 \pm 1.52$ & $10.66 \pm 1.15$ & $06.66 \pm 1.15$ & $10.00 \pm 0.00$ & $04.00 \pm 1.00$ & $05.00 \pm 0.00$ & $05.33 \pm 1.52$ & NA & NA & NA & $18.66 \pm 1.15$ \\
\hline 02 & Aegle marmelos $\mathrm{L}$. & $\mathrm{L}$ & $07.00 \pm 0.00$ & $05.00 \pm 1.00$ & $07.33 \pm 1.52$ & $05.00 \pm 1.00$ & $08.00 \pm 1.00$ & $05.33 \pm 1.52$ & $10.00 \pm 1.00$ & $05.00 \pm 0.00$ & $06.00 \pm 0.00$ & $04.00 \pm 0.00$ & NA & $21.00 \pm 1.00$ \\
\hline 03 & Allium cepa $\mathrm{L}$. & $\mathrm{B}$ & $05.00 \pm 1.00$ & NA & $06.33 \pm 1.52$ & $05.66 \pm 1.15$ & $30.00 \pm 1.00$ & $06.33 \pm 1.52$ & $07.00 \pm 1.00$ & $05.33 \pm 1.52$ & $05.00 \pm 0.00$ & NA & NA & 15. $66 \pm 0.57$ \\
\hline 04 & Allium sativam $\mathrm{L}$. & $\mathrm{B}$ & $06.66 \pm 0.57$ & $05.33 \pm 0.57$ & $10.66 \pm 1.15$ & $06.00 \pm 0.00$ & $12.00 \pm 0.00$ & $04.66 \pm 0.57$ & $05.66 \pm 1.15$ & $05.33 \pm 0.57$ & NA & NA & NA & 18. $66 \pm 0.57$ \\
\hline 05 & Aloe vera $\mathrm{L}$. & $\mathrm{L}$ & $10.66 \pm 0.57$ & $04.00 \pm 0.00$ & $06.33 \pm 1.52$ & $07.00 \pm 0.00$ & $07.00 \pm 1.00$ & $06.33 \pm 1.52$ & $05.00 \pm 0.00$ & $04.00 \pm 1.00$ & $06.00 \pm 0.00$ & NA & NA & 21. $66 \pm 0.57$ \\
\hline 06 & Amaranthus spinosus L. & $\mathrm{L}$ & $04.33 \pm 1.15$ & NA & $07.00 \pm 0.00$ & $05.00 \pm 0.00$ & $05.00 \pm 1.00$ & $05.00 \pm 0.00$ & $06.66 \pm 1.57$ & $05.66 \pm 0.57$ & $05.00 \pm 0.00$ & NA & NA & $16.00 \pm 0.00$ \\
\hline 07 & Annona reticulata L. & $\mathrm{L}$ & $05.00 \pm 0.00$ & $04.66 \pm 0.57$ & $06.33 \pm 1.15$ & $05.00 \pm 0.00$ & $10.66 \pm 1.57$ & $05.00 \pm 1.00$ & $14.00 \pm 1.00$ & $05.00 \pm 0.00$ & $05.66 \pm 1.52$ & $04.66 \pm 1.57$ & NA & 22. $33 \pm 1.52$ \\
\hline 08 & Annona squamosa $\mathrm{L}$. & $\mathrm{L}$ & $07.66 \pm 1.57$ & $05.33 \pm 1.52$ & $06.00 \pm 1.00$ & $05.66 \pm 0.57$ & $14.66 \pm 0.57$ & $06.66 \pm 1.52$ & $12.66 \pm 1.52$ & $06.66 \pm 0.57$ & $06.66 \pm 1.52$ & NA & NA & $17.00 \pm 1.00$ \\
\hline 09 & Argemone mexicana $\mathrm{L}$. & $\mathrm{L}$ & $05.33 \pm 1.52$ & NA & $08.00 \pm 1.52$ & $04.66 \pm 0.57$ & $05.66 \pm 1.52$ & NA & $04.33 \pm 1.52$ & $04.66 \pm 1.52$ & $13.33 \pm 1.52$ & NA & NA & 15. $33 \pm 1.52$ \\
\hline 10 & Azadirachta indica A.Juss. & s. $\mathrm{L}$ & $06.00 \pm 1.57$ & $04.33 \pm 1.52$ & $04.00 \pm 1.00$ & $04.66 \pm 1.57$ & $06.33 \pm 1.52$ & $05.66 \pm 1.57$ & $06.00 \pm 1.00$ & $06.66 \pm 0.57$ & $04.33 \pm 1.52$ & NA & NA & 13. $66 \pm 0.57$ \\
\hline 11 & Bergera koenigii L. & $\mathrm{L}$ & $07.66 \pm 1.52$ & $05.66 \pm 1.52$ & $10.00 \pm 0.00$ & $06.66 \pm 1.52$ & $08.33 \pm 0.57$ & $06.66 \pm 1.52$ & $07.00 \pm 0.00$ & $05.33 \pm 0.57$ & $04.66 \pm 1.52$ & NA & NA & 17. $33 \pm 1.52$ \\
\hline 12 & $\begin{array}{l}\text { Butea monosperma (Lam.) } \\
\text { Taub. }\end{array}$ & $\mathrm{L}$ & $05.33 \pm 1.15$ & $04.33 \pm 0.57$ & $06.33 \pm 0.57$ & $05.66 \pm 1.52$ & $13.33 \pm 0.57$ & $09.33 \pm 1.52$ & $10.33 \pm 1.52$ & $06.33 \pm 1.52$ & $04.66 \pm 1.52$ & NA & NA & $22.00 \pm 0.00$ \\
\hline 13 & Cajanus cajan (L.)Mill. & $\mathrm{L}$ & $05.66 \pm 0.57$ & 05.00 .0 .00 & $06.66 \pm 1.57$ & $05.66 \pm 1.57$ & $07.66 \pm 0.57$ & $05.33 \pm 0.57$ & $06.66 \pm 0.57$ & $05.00 \pm 1.00$ & $05.66 \pm 0.57$ & NA & NA & 14. $66 \pm 1.15$ \\
\hline 14 & Calotropis gigantea $\mathrm{L}$. & $\mathrm{L}$ & $06.33 \pm 0.57$ & $05.66 \pm 1.57$ & $07.33 \pm 0.57$ & $06.66 \pm 0.57$ & $07.66 \pm 1.57$ & $06.66 \pm 0.57$ & $07.00 \pm 1.00$ & $05.66 \pm 1.57$ & $06.66 \pm 1.57$ & NA & NA & 26. $66 \pm 0.57$ \\
\hline 15 & Carica papaya $\mathrm{L}$ & $\mathrm{L}$ & $05.33 \pm 1.15$ & $04.66 \pm 1.57$ & $04.33 \pm 0.57$ & $04.66 \pm 1.52$ & $06.33 \pm 0.57$ & $05.33 \pm 0.57$ & $05.66 \pm 1.52$ & $05.66 \pm 1.52$ & $06.33 \pm 0.57$ & NA & NA & $13.00 \pm 0.00$ \\
\hline 17 & Celosia argentea $\mathrm{L}$. & $\mathrm{S}$ & $07.00 \pm 0.00$ & $05.33 \pm 0.57$ & $11.00 \pm 0.00$ & $06.00 \pm 0.00$ & $07.66 \pm 0.57$ & $06.00 \pm 0.00$ & $08.00 \pm 0.00$ & $05.00 \pm 0.00$ & $05.66 \pm 0.57$ & NA & NA & 12. $33 \pm 1.52$ \\
\hline 18 & Citrus medica L. & $\mathrm{L}$ & $06.66 \pm 0.57$ & $05.33 \pm 0.57$ & $08.66 \pm 0.57$ & $05.66 \pm 0.57$ & $07.33 \pm 0.57$ & $05.33 \pm 1.15$ & $05.3 \pm 1.15$ & $04.33 \pm 0.57$ & 12. $3 \pm 1.15$ & NA & NA & 19. $66 \pm 0.57$ \\
\hline 19 & $\begin{array}{l}\text { Coccinia indica Wt. \& } \\
\text { Arn. }\end{array}$ & $\mathrm{L}$ & $06.66 \pm 1.52$ & $05.00 \pm 0.00$ & $06.33 \pm 1.15$ & $04.33 \pm 1.15$ & $11.00 \pm 0.00$ & $07.33 \pm 1.15$ & $08.33 \pm 1.15$ & $07.00 \pm 0.00$ & $05.66 \pm 0.57$ & NA & NA & 16. $66 \pm 0.57$ \\
\hline 20 & Corchorus oleterius L. & $\mathrm{S}$ & $06.66 \pm 0.57$ & $04.33 \pm 1.15$ & $08.66 \pm 0.57$ & $05.6 \pm 1.52$ & $17.66 \pm 1.52$ & $05.3 \pm 1.15$ & $14.66 \pm 0.57$ & $05.6 \pm 1.52$ & 12. $3 \pm 1.15$ & $07.66 \pm 1.57$ & NA & 22. $33 \pm 1.52$ \\
\hline 21 & Coriandrum sativam $\mathrm{L}$. & A & $05.3 \pm 1.15$ & $04.00 \pm 0.00$ & $05.33 \pm 0.57$ & $04.3 \pm 1.15$ & $06.33 \pm 0.57$ & 04. $0 \pm 0.00$ & $05.33 \pm 1.15$ & $04.33 \pm 0.57$ & $04.66 \pm 1.52$ & NA & NA & $15.33 \pm 1.52$ \\
\hline 22 & $\begin{array}{l}\text { Cryptolepis buchananii } \\
\text { Roem\&Schult. }\end{array}$ & A & $05.00 \pm 0.00$ & $05.33 \pm 1.15$ & $06.33 \pm 1.15$ & $05.00 \pm 0.00$ & $07.00 \pm 0.00$ & $06.00 \pm 0.00$ & $07.66 \pm 1.52$ & $05.00 \pm 0.00$ & $05.00 \pm 0.00$ & NA & NA & 17. $33 \pm 1.52$ \\
\hline 23 & Curcuma longa $\mathrm{L}$. & $\mathrm{R}$ & $06.33 \pm 0.57$ & $09.66 \pm 1.52$ & $06.66 \pm 1.52$ & $05.33 \pm 0.57$ & $05.33 \pm 1.15$ & $05.66 \pm 1.52$ & $08.33 \pm 1.15$ & $05.33 \pm 0.57$ & $05.66 \pm 1.52$ & NA & NA & 19. $66 \pm 0.57$ \\
\hline 24 & Dalbergia sisso Roxb. & $\mathrm{L}$ & $07.66 \pm 1.52$ & $06.66 \pm 0.57$ & $07.33 \pm 1.52$ & $06.00 \pm 0.00$ & $08.66 \pm 0.57$ & $07.33 \pm 1.52$ & $08.66 \pm 0.57$ & $06.66 \pm 1.52$ & $05.00 \pm 1.00$ & NA & NA & 27. $33 \pm 1.52$ \\
\hline 25 & Datura metel L. & $\mathrm{L}$ & $07.66 \pm 0.57$ & $05.66 \pm 1.57$ & $07.66 \pm 0.57$ & $04.00 \pm 0.00$ & $11.66 \pm 1.52$ & $06.66 \pm 0.57$ & $07.00 \pm 0.00$ & $04.66 \pm 0.57$ & $07.00 \pm 0.00$ & $05.66 \pm 0.57$ & NA & $19.33 \pm 1.52$ \\
\hline 26 & Emblica officinalis Gaertn. & 1. $\mathrm{L}$ & $07.66 \pm 0.57$ & $05.66 \pm 0.57$ & $05.00 \pm 0.00$ & $05.66 \pm 0.57$ & $12.66 \pm 0.57$ & $05.00 \pm 1.00$ & $06.33 \pm 1.15$ & $07.66 \pm 1.52$ & $05.66 \pm 0.57$ & NA & NA & 17. $66 \pm 1.15$ \\
\hline 27 & Euphorbia tirucalli $\mathrm{L}$. & $\mathrm{L}$ & $05.66 \pm 1.57$ & $05.66 \pm 0.57$ & $08.33 \pm 1.52$ & $06.66 \pm 1.52$ & $06.00 \pm 1.00$ & $05.66 \pm 1.52$ & $12.33 \pm 1.52$ & $04.33 \pm 1.52$ & $06.66 \pm 1.57$ & NA & NA & 18. $33 \pm 1.52$ \\
\hline 28 & Ficus racemosa $\mathrm{L}$ & $\mathrm{L}$ & $05.66 \pm 0.57$ & $05.66 \pm 0.57$ & $06.66 \pm 0.57$ & $06.66 \pm 1.52$ & $10.33 \pm 1.52$ & $08.66 \pm 1.52$ & $12.66 \pm 1.57$ & $07.66 \pm 0.57$ & $05.00 \pm 0.00$ & NA & NA & $28.33 \pm 1.52$ \\
\hline 29 & $\begin{array}{l}\text { Gymnosporia montana } \\
\text { (Roth)Benth }\end{array}$ & $\mathrm{L}$ & $04.66 \pm 0.57$ & $04.66 \pm 0.57$ & $07.00 \pm 0.00$ & $05.33 \pm 1.52$ & $12.66 \pm 1.57$ & $06.66 \pm 1.57$ & $07.66 \pm 0.57$ & $07.33 \pm 1.15$ & $05.66 \pm 0.57$ & NA & NA & 17. $66 \pm 1.15$ \\
\hline 30 & Hibiscus rosa-sinensis L. & $\mathrm{F}$ & $05.66 \pm 0.57$ & $04.66 \pm 1.52$ & $07.66 \pm 1.57$ & $05.66 \pm 0.57$ & $06.00 \pm 0.00$ & $05.66 \pm 1.52$ & $07.66 \pm 0.57$ & $06.00 \pm 0.00$ & $04.66 \pm 0.57$ & NA & NA & 20. \\
\hline 31 & $\begin{array}{l}\text { Hyptis suoveolens } \\
\text { (L.)Poit. }\end{array}$ & $\mathrm{L}$ & $05.33 \pm 0.57$ & $05.66 \pm 1.52$ & $08.33 \pm 1.15$ & $06.00 \pm 0.00$ & $08.33 \pm 1.15$ & $06.66 \pm 1.52$ & $07.66 \pm 1.52$ & $06.33 \pm 1.15$ & $05.33 \pm 0.57$ & NA & NA & $18.00 \pm 0.00$ \\
\hline 33 & $\begin{array}{l}\text { Jatropha glandulifera } \\
\text { Roxb. }\end{array}$ & $\mathrm{L}$ & $06.66 \pm 1.52$ & $06.66 \pm 1.57$ & $09.33 \pm 0.57$ & $05.66 \pm 1.57$ & $08.66 \pm 0.57$ & $06.66 \pm 1.57$ & $10.66 \pm 0.57$ & $05.00 \pm 0.00$ & $06.66 \pm 1.52$ & NA & NA & 18. $66 \pm 1.15$ \\
\hline 34 & Lantana camara $\mathrm{L}$. & $\mathrm{L}$ & NA & NA & $08.33 \pm 1.15$ & $04.00 \pm 0.00$ & $04.33 \pm 1.15$ & NA & $06.00 \pm 0.00$ & $05.66 \pm 0.57$ & $05.66 \pm 1.52$ & NA & NA & $18.00 \pm 0.00$ \\
\hline 35 & Lawsonia inermis Linn. & $\mathrm{L}$ & $04.66 \pm 1.57$ & $\mathrm{NA} 33 \pm 1.15$ & $05.00 \pm 1.00$ & $05.33 \pm 1.52$ & $13.33 \pm 1.52$ & $06.33 \pm 0.57$ & NA & $06.00 \pm 0.00$ & $04.66 \pm 0.57$ & NA & NA & 17. $33 \pm 1.52$ \\
\hline 36 & $\begin{array}{l}\text { Lycopersicon esculentum } \\
\text { L. }\end{array}$ & $\mathrm{L}$ & $07.00 \pm 0.00$ & $05.66 \pm 1.52$ & $08.66 \pm 1.52$ & $05.33 \pm 1.15$ & $09.66 \pm 1.52$ & $06.33 \pm 1.15$ & $09.66 \pm 1.52$ & $05.33 \pm 0.57$ & $06.00 \pm 0.00$ & NA & NA & 19. $33 \pm 1.5$ \\
\hline 37 & Mangifera indica Linn. & $\mathrm{L}$ & $07.33 \pm 0.57$ & $06.66 \pm 1.57$ & $06.33 \pm 1.15$ & $06.66 \pm 0.57$ & $06.33 \pm 1.15$ & $05.66 \pm 1.52$ & $06.66 \pm 1.57$ & $04.33 \pm 1.15$ & $05.66 \pm 1.57$ & NA & NA & 25.66 \\
\hline 38 & Mentha viridis $\mathrm{L}$. & A & $04.66 \pm 0.57$ & $05.66 \pm 0.57$ & NA & $04.33 \pm 0.57$ & $04.66 \pm 1.57$ & NA & $05.66 \pm 1.57$ & $04.66 \pm 1.52$ & $06.66 \pm 0.57$ & NA & NA & $15.50 \pm 0.00$ \\
\hline 39 & $\begin{array}{l}\text { Milletia pinnata }(\mathrm{L} .) \\
\text { Panigrahi }\end{array}$ & $\mathrm{L}$ & $11.00 \pm 1.00$ & $06.66 \pm 0.57$ & $11.33 \pm 1.52$ & $09.66 \pm 1.57$ & $07.00 \pm 1.00$ & $05.66 \pm 1.52$ & $09.66 \pm 1.57$ & $09.66 \pm 0.57$ & NA & NA & NA & $20.66 \pm 0.57$ \\
\hline 40 & Momordica charantia $\mathrm{L}$. & $\mathrm{L}$ & $04.66 \pm 1.57$ & $04.00 \pm 1.00$ & $05.33 \pm 0.57$ & $04.33 \pm 1.52$ & $06.33 \pm 1.52$ & $05.33 \pm 0.57$ & $05.33 \pm 0.57$ & $05.00 \pm 1.00$ & $05.66 \pm 1.57$ & NA & NA & 16. $66 \pm 1.15$ \\
\hline 41 & Nerium odorum Solander. & $\mathrm{L}$ & $06.33 \pm 1.15$ & $05.66 \pm 0.57$ & $05.33 \pm 1.52$ & $05.66 \pm 1.52$ & $07.00 \pm 1.00$ & $06.33 \pm 1.52$ & $06.66 \pm 1.57$ & $05.66 \pm 1.52$ & $05.66 \pm 1.52$ & NA & NA & $14.00 \pm 0.00$ \\
\hline 42 & Ocimum sanctum $\mathrm{L}$. & A & $06.33 \pm 1.52$ & $05.66 \pm 0.57$ & $09.66 \pm 1.57$ & $04.33 \pm 1.52$ & $05.33 \pm 0.57$ & $04.66 \pm 1.57$ & $05.33 \pm 0.57$ & $05.66 \pm 0.57$ & $04.00 \pm 1.00$ & NA & NA & 15. $33 \pm 1.52$ \\
\hline 43 & Piper nigrum $\mathrm{L}$. & $\mathrm{S}$ & $05.66 \pm 0.57$ & $05.00 \pm 1.00$ & $07.33 \pm 1.52$ & $05.66 \pm 1.57$ & $06.00 \pm 1.00$ & $06.33 \pm 0.57$ & $06.66 \pm 0.57$ & $04.66 \pm 1.52$ & $05.00 \pm 1.00$ & NA & NA & 28. $66 \pm 1.15$ \\
\hline 44 & Plumbago zeylanica $\mathrm{L}$. & $\mathrm{L}$ & $10.66 \pm 0.57$ & $04.00 \pm 1.00$ & $08.33 \pm 1.52$ & $04.6 \pm 1.57$ & $07.00 \pm 1.00$ & $04.33 \pm 1.52$ & $09.66 \pm 1.57$ & $05.66 \pm 1.57$ & $06.00 \pm 1.00$ & NA & NA & $24.00 \pm 0.00$ \\
\hline 45 & Ricinus communis L. & $\mathrm{S}$ & $05.33 \pm 0.57$ & $04.66 \pm 0.57$ & $04.33 \pm 0.57$ & $04.33 \pm 0.57$ & $07.00 \pm 0.00$ & $05.66 \pm 1.57$ & $06.66 \pm 0.57$ & $05.33 \pm 1.15$ & $04.66 \pm 0.57$ & NA & NA & $15.33 \pm 1.52$ \\
\hline 46 & Santalum album L. & $\mathrm{L}$ & $04.33 \pm 1.52$ & $04.00 \pm 0.00$ & $05.33 \pm 1.52$ & $04.66 \pm 0.57$ & $07.33 \pm 1.52$ & $06.66 \pm 1.57$ & $06.00 \pm 0.00$ & $05.33 \pm 1.52$ & $04.33 \pm 1.52$ & NA & NA & $10.33 \pm 1.52$ \\
\hline 47 & $\begin{array}{l}\text { Senna auriculata (L.) } \\
\text { Roxb. }\end{array}$ & $\mathrm{F}$ & $06.66 \pm 0.57$ & $05.33 \pm 1.52$ & $07.66 \pm 0.57$ & $06.66 \pm 1.57$ & $10.00 \pm 0.00$ & $06.66 \pm 0.57$ & $08.66 \pm 1.52$ & $05.66 \pm 0.57$ & $05.00 \pm 0.00$ & NA & NA & 17. $66 \pm 1.15$ \\
\hline 48 & Senna tora $\mathrm{L}$. & $\mathrm{L}$ & $05.66 \pm 1.57$ & $05.66 \pm 1.57$ & $08.66 \pm 1.52$ & $06.33 \pm 1.52$ & $11.66 \pm 0.57$ & $07.33 \pm 1.52$ & $07.33 \pm 1.52$ & $06.66 \pm 0.57$ & $05.66 \pm 1.52$ & NA & NA & $18.50 \pm 0.00$ \\
\hline 49 & Solanum nigrum $\mathrm{L}$. & $\mathrm{L}$ & $06.00 \pm 0.00$ & $07.66 \pm 0.57$ & $08.66 \pm 1.57$ & $07.66 \pm 1.52$ & $12.66 \pm 0.57$ & $08.66 \pm 1.57$ & $11.33 \pm 0.57$ & $08.00 \pm 0.00$ & $10.66 \pm 1.57$ & NA & NA & 14. $66 \pm 1.15$ \\
\hline 50 & Sterculia foetida $\mathrm{L}$. & $\mathrm{S}$ & $11.66 \pm 1.57$ & $05.66 \pm 1.52$ & 14. $00 \pm 0.00$ & $05.66 \pm 0.57$ & $08.33 \pm 1.52$ & $05.33 \pm 1.52$ & $10.66 \pm 1.57$ & $05.33 \pm 0.57$ & $05.33 \pm 1.52$ & NA & NA & $20.00 \pm 0.00$ \\
\hline 51 & $\begin{array}{l}\text { Semecarpus anacardium } \\
\text { L. }\end{array}$ & B & $08.66 \pm 0.57$ & $04.66 \pm 1.57$ & $08.66 \pm 1.52$ & $05.66 \pm 0.57$ & $07.33 \pm 1.52$ & $05.66 \pm 1.57$ & $06.33 \pm 1.52$ & $05.66 \pm 1.57$ & $05.66 \pm 0.57$ & NA & NA & 16. $66 \pm 1.15$ \\
\hline 52 & Tamarindus indica Linn. & $\mathrm{L}$ & $04.66 \pm 0.57$ & NA & 11. $00 \pm 0.00$ & $06.33 \pm 1.52$ & $10.66 \pm 1.57$ & $07.00 \pm 0.00$ & $09.00 \pm 0.00$ & $06.33 \pm 1.52$ & $06.33 \pm 0.57$ & NA & NA & \\
\hline 53 & Tectona grandis $\mathrm{L}$. & $\mathrm{L}$ & $06.00 \pm 0.00$ & $05.00 \pm 33 \pm 0.57$ & $708.33 \pm 1.52$ & $05.66 \pm 0.57$ & $10.33 \pm 0.57$ & $05.66 \pm 0.57$ & $10.33 \pm 0.57$ & $06.66 \pm 1.57$ & $05.66 \pm 0.57$ & NA & NA & 18. $66 \pm 1.15$ \\
\hline 54 & $\begin{array}{l}\text { Tinospora cordifolia } \\
\text { (Willd.)J.Hook\&Thoms }\end{array}$ & $\mathrm{L}$ & $05.66 \pm 1.57$ & $04.66 \pm 1.57$ & $04.00 \pm 0.00$ & $04.33 \pm 1.52$ & $10.66 \pm 1.57$ & $06.66 \pm 1.57$ & $05.33 \pm 1.52$ & $05.66 \pm 0.57$ & $05.33 \pm 1.52$ & NA & NA & $18.00 \pm 0.00$ \\
\hline 55 & $\begin{array}{l}\text { Tephrosia purpurea }(\mathrm{L} .) \\
\text { Pers. }\end{array}$ & $\mathrm{L}$ & $05.00 \pm 0.00$ & $04.33 \pm 1.52$ & $08.66 \pm 0.57$ & $05.66 \pm 1.57$ & $08.33 \pm 1.52$ & $05.33 \pm 1.52$ & $07.66 \pm 0.57$ & $04.66 \pm 1.57$ & $05.33 \pm 1.15$ & NA & NA & 18. 66 \\
\hline 56 & Thevetia nerrifolia Juss. & $\mathrm{L}$ & $06.00 \pm 0.00$ & $04.66 \pm 0.57$ & $06.66 \pm 1.57$ & $05.33 \pm 1.52$ & $10.66 \pm 0.57$ & $05.33 \pm 1.52$ & $07.33 \pm 0.57$ & $05.66 \pm 0.57$ & $04.66 \pm 1.57$ & NA & NA & 22. $66 \pm 1.15$ \\
\hline 57 & Tribulus terrestris $\mathrm{L}$. & A & $05.33 \pm 0.57$ & $04.33 \pm 1.52$ & $06.66 \pm 1.57$ & $05.33 \pm 1.15$ & $12.66 \pm 1.57$ & $05.66 \pm 0.57$ & $07.33 \pm 1.15$ & $04.66 \pm 0.57$ & $04.33 \pm 1.52$ & NA & NA & 24. $66 \pm 0.57$ \\
\hline 58 & Tridax procumbens Linn. & A & $04.33 \pm 1.52$ & NA & $04.00 \pm 0.00$ & NA & $04.66 \pm 0.57$ & $07.66 \pm 1.57$ & $05.33 \pm 1.52$ & $06.66 \pm 1.57$ & NA & NA & NA & $18.00 \pm 0.00$ \\
\hline 59 & Vitex negundo $\mathrm{L}$. & $\mathrm{L}$ & $05.66 \pm 1.57$ & $05.00 \pm 1.00$ & $06.33 \pm 0.57$ & $06.66 \pm 1.52$ & $14.66 \pm 1.57$ & $08.00 \pm 0.00$ & $12.66 \pm 1.57$ & $07.66 \pm 0.57$ & $05.33 \pm 0.57$ & NA & NA & 28. $66 \pm 1.15$ \\
\hline 60 & Zingiber officinale Rosce. & $\mathrm{R}$ & $05.33 \pm 0.57$ & $09.33 \pm 1.52$ & $08.00 \pm 1.00$ & $06.00 \pm 1.00$ & $13.33 \pm 1.52$ & $06.33 \pm 1.52$ & $06.00 \pm 1.00$ & $05.33 \pm 1.15$ & NA & NA & NA & $15.00 \pm 0.00$ \\
\hline 61 & Zizyphus jujuba Lam. & $\mathrm{B}$ & $05.00 \pm 1.00$ & $04.00 \pm 66 \pm 1.57$ & 704. $33 \pm 1.52$ & NA & $07.00 \pm 1.00$ & NA & $07.33 \pm 1.15$ & $05.33 \pm 1.52$ & $\mathrm{NA}$ & NA & NA & $21.33 \pm 1.52$ \\
\hline
\end{tabular}


skin diseases in Hyderabad Karnataka region were subjected to antidermatophytic screening against Trichophyton rubrum (MTCC 1344) in Pet ether, chloroform, ethyalacetate, methanol and aqueous extracts of each plant were tested for their antifungal activity using agar well diffusion method at a sample concentration of $5 \& 2.5 \mathrm{mg} / \mathrm{ml}$. Out of 61 plants, 18 exhibited very effective antidermatophytic activity in methanolic extract i.e., Allium sativam Linn., Annona reticulata L., Annona squamosa L., Argemone mexicana L., Butea monosperma, Ceasalpinia bonducella, Citrus medica L., Corchorus oleterius L., Emblica officinalis, Euphorbia tirucalli L., Ficus racemosa L., Gymnosporia montana, Lawsonia inermis Linn., Solanum nigrum L., Sterculia foetida L., Tribulus terrestris L., Vitex negundo L., and Zingiber officinale, The percentage of solvent dissolving extracts and family ratio were shown in figure. $2 \& 3$.

Followed by effective activity observed in 13 plants of different solvent extracts, i.e., Coccinia indica, Datura metel, Senna auriculata, Senna tora, Tectona grandis, Tinospora cordifolia, Thevetia nerrifolia (ethyl acetate), Achyranthes aspera, Bergera koenigii, Celosia argentea, Tamarindus indica (chloroform) Aloe vera, Milletia pinnata (petroleum ether). Whereas the weak activity observed in 04 plants, i.e., Lantana camara, Mentha viridis, Tridax procumbens and Zizyphus jujuba. There was no inhibition recorded from the negative control (DMSO), while the standard drug, Ketoconazole significantly inhibited ( $28.66 \pm 1.15$ to $12.33 \pm 1.52 \mathrm{~mm})$ the growth of the test dermatophyte.

The maximum activity of very effective and effective plants extracts shown in figure $5 \& 6$ respectively. The minimum inhibitory concentrations of very effective 18 plants were determined, among the 18 plants extracts 04 i.e., Allium cepa Linn., Euphorbia tirucalli L., Lawsonia inermis, Tribulus terrestris L., were showed highest MIC at $0.31 \mathrm{mg} / \mathrm{ml}$ conc. (Figure 4).

In the present report the effective activity observed in 18 plants of methanolic extract concentrations of between 05 and $2.5 \mathrm{mg} / \mathrm{ml}$, this result is in line with the work of Shinkafi and Manga, (2011), who reported that the aqueous and organic leaf extracts of Mitracarpus scaber and Pergularia tomentosa exhibited significant anti-fungal activities against T. mentagrophytes, $T$. rubrum and $M$. gypseum at extracts concentrations of between 80 and $160 \mathrm{mg} / \mathrm{ml}$. The activities of the methanol extract were higher, though not significant $(\mathrm{P}>0.05)$ when compared with the aqueous extract. The reason for this slight difference may be attributed to the solubility level of the phytoconstituents in the ext-racting solvents. It means that the methanol dissolved more of more of the active ingredients than aqueous solvent.

This reason is supported by Cowan (1999), who reported that organic solvent were better extraction solvent over water. The response of dermatophyte to treatment with various plant extracts varied from solvent extract to solvent extract; nevertheless it was shown to be dose dependent as greater inhibition of growth was observed as the concentrations of the extracts increased. This is supported by the work of (Bharti and Vidyasagar, 2012; Shivakumar and Vidyasagar, 2014), where they revealed that, ethyl acetate extract showed antimycotic activity against $T$. rubrum and T. mentagrophytes.

Chloroform and methanol extracts showed activity at concentration of $50 \mathrm{mg} / \mathrm{ml}$ where as ethyl acetate showed activity at high concentration of $150 \mathrm{mg} / \mathrm{ml}$. The aqueous extracts of Allium sativum and Ocimum sanctum at $10 \%$ conc. were more pronounced antifungal properties against the dermatophytic fungus Microsporum gypseum. (N.C. Sowjanya and C.Manohara Chary, 2012).

The MIC of 18 effective plants of methanolic extracts showed different values was recorded (Figure.4). The similar to that from the work of Ali-Shtayeh and Abu-Ghdeib (1999), who reported that aqueous extracts of 22 plants recorded wide variations in their MIC values against Microsporum canis, Trichophyton mentagrophytes and Trichophyton violaceum. This could be attributed to the variations in the phytochemical properties of the plants and differences among the fungal species.

In the present report the weak activity was observed in 04 plants, i.e., Lantana camara, Mentha viridis, Tridax procumbens and Zizyphus jujuba. Whereas in previous report Lantana camara was recorded as a most active in the 61plants series against pathogenic bacteria (V. Prashanth Kumar, Neelam S. 2006). This could probably suggest that certain phytochemicals exhibit their antifungal action only with other phytoconstituents in antibacterial. According to the previous similar report among the 21 plant methanolic extracts showed effective activity against two bacteria, but 20 plants were shown activity against two fungi (R. S. Taylor et al., 1995).

The methanolic and ethyalacetate solvent extracts were very effective and effective respectively in the present study. The similar results reported by Mehmood Z et al., (1999) ethanol extracts showed an inhibitory effect against the three Trichophyton spp. Preliminary phytochemical investigation revealed the presence of saponins, glycosides, tannins, alkaloids and flavonoids, as indicated in Table 2.

The crude successive extracts of 61 traditional medicinal plants were qualitatively screened for the occurrence of various secondary metabolites such as phenols (Lead acetate test), flavonoids ( $\mathrm{NaOH}$ test), tannins (Ferric chloride test), alkaloids (Dragendroff's test), Saponins Foam test), glycosides (KellerKilliani test). The reactions with these reagents have shown the presence of metabolites and record in the Table -4 .

The present study suggests that the effective extracts of these plants is a potential source of natural antifungal agents. After this screening experiment, further work should be performed to describe the antifungal activities in more detail as well as their activity in-vivo. In addition, phytochemical studies will be necessary to isolate the active constituents and evaluate the antidermatophytic activities against a wide range of fungi population. 
Table 2: Preliminary phytochemical screening for the detection of secondary metabolites in 18 effective folkoric plant extracts.

\begin{tabular}{|c|c|c|c|c|c|c|}
\hline \multirow{2}{*}{ Plant name and Family } & \multicolumn{6}{|c|}{ Phyto constituents } \\
\hline & Phenols & Flavonoids & Tannins & Alkaloids & Saponins & Glycosides \\
\hline Allium sativam Linn. (Liliaceae) & --+ & --+ & --- & ++- & ++- & +++ \\
\hline Annona reticulata L. (Annonaceae) & ++- & +-- & ++- & --+ & --- & --+ \\
\hline Annona squamosa L. (Annonaceae) & +++ & ++- & --+ & --+ & -- & -- \\
\hline Argemone mexicana L.(Papaveraceae) & -++ & --+ & +++ & --+ & --+ & --- \\
\hline Butea monosperma (Lam) Taub. (Fabaceae) & --+ & --+ & +++ & ++- & --- & --- \\
\hline Ceasalpinia bonducella (L.) Flem. (Ceasalpiniaceae) & +++ & +++ & ++- & ++- & ++- & --+ \\
\hline Citrus limon (Rutaceae) & --+ & -++ & +++ & +++ & --+ & --- \\
\hline Corchorus capsularis L. (Tiliaceae) & ++- & ++- & ++- & --- & -- & --+ \\
\hline Emblica officinalis Gaertn. (Euphorbiaceae) & --+ & +++ & ++- & +++ & --+ & --+ \\
\hline Euphorbia tirucalli L. (Euphorbiaceae) & +++ & ++- & --+ & +++ & --+ & ++- \\
\hline Ficus racemosa L.(Moraceae) & --+ & +++ & --+ & ++- & --- & -- \\
\hline Lawsonia inermis Linn. (Lythraceae) & +++ & +++ & --+ & ++- & --+ & --+ \\
\hline Solanum nigrum L. (Solanaceae) & --+ & +++ & +++ & --+ & -- & --+ \\
\hline Sterculia foetida L (Sterculaceae) & +++ & ++ & ++ & ++- & --+ & --- \\
\hline Tribulus terrestris L (Zygophyllaceae) & --+ & --+ & ++- & +++ & --+ & -- \\
\hline Vitex negudu (Verbenaceae) & ++- & +++ & +++ & --+ & -- & --+ \\
\hline Zingiber officinale Rosce. (Zingiberaceae) & ++- & ++- & ++- & ++- & --+ & --. \\
\hline
\end{tabular}

+ present, - absent, +++ strongly present, ---strongly absent.

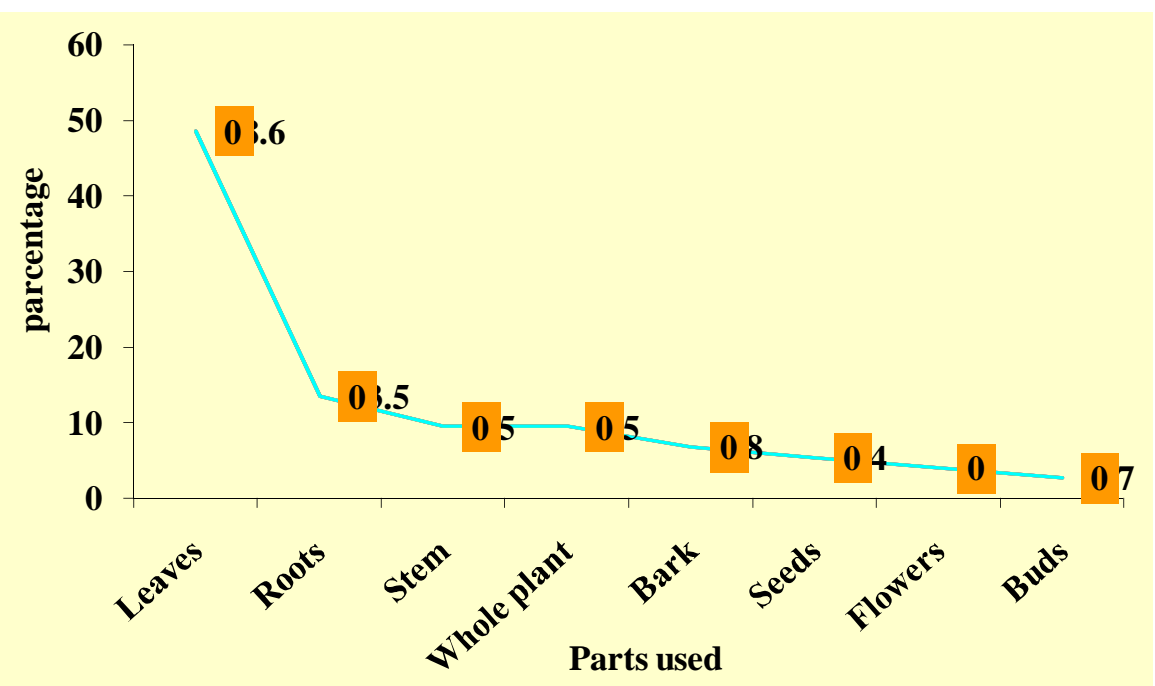

Fig. 1: 61 medicinal plant parts used against T. rubrum.

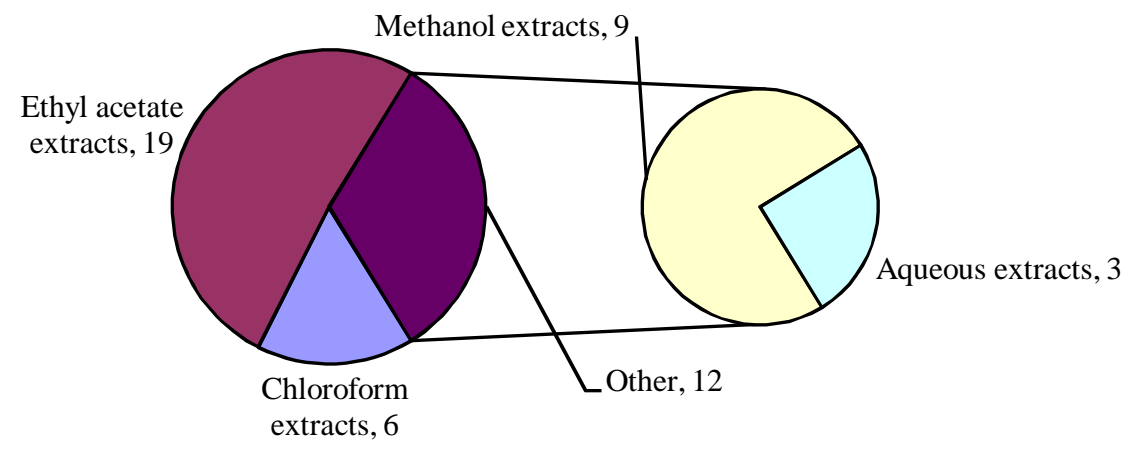

Fig. 2: Effective extracts of 18 medicinal plants against T. rubrum. 


\begin{tabular}{|lll|}
\hline $\mathbf{\square}$ Euphorbiaceae & $\mathbf{\square}$ Fabaceae & $\mathbf{\square}$ Caesalpiniceae \\
$\mathbf{\square}$ Amarathaceae & $\mathbf{\square}$ Lamiaceae & $\mathbf{\square}$ Liliaceae \\
$\mathbf{\square}$ Rutaceae & $\mathbf{\square}$ Solanaceae & $\mathbf{\square}$ Verbenaceae \\
$\mathbf{\square}$ Anacardiaceae & $\mathbf{\square}$ Annonaceae & $\mathbf{\square}$ Apocynaceae \\
$\mathbf{\square}$ Asclepiadaceae & $\mathbf{\square}$ Cucurbitaceae & $\mathbf{\square}$ Zingiberaceae \\
$\mathbf{\square}$ 18families & & \\
\hline
\end{tabular}

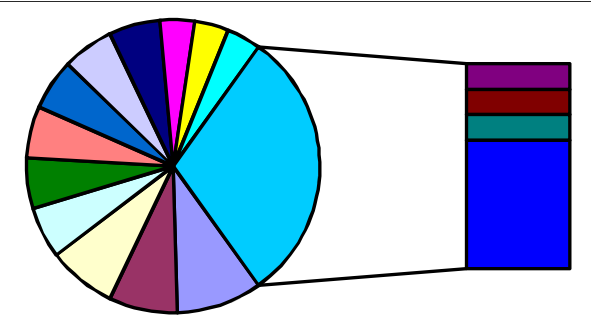

Fig. 3: Family wise percentage of medicinal plants against T. rubrum.

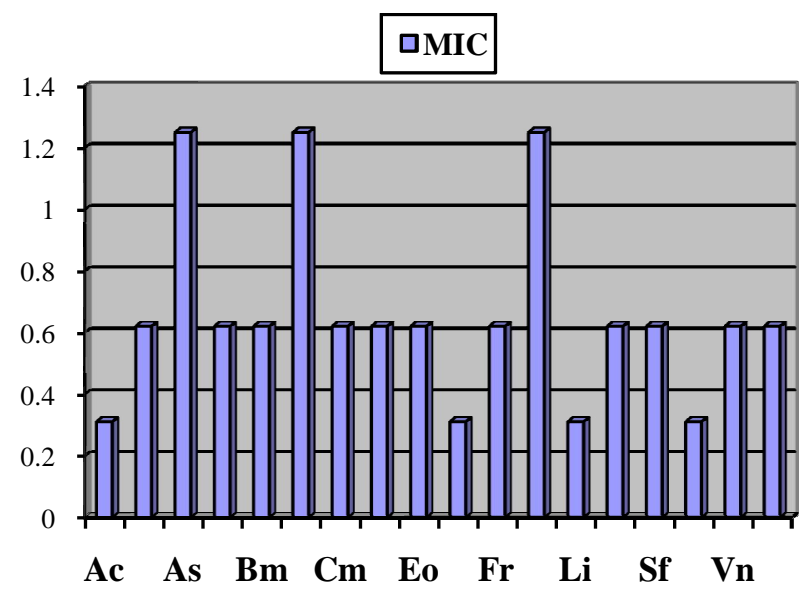

Fig. 4: Minimum Inhibitory Concentration $(\mathrm{mg} / \mathrm{ml})$ of 18 effective medicinal plants of methanolic extracts against T. rubrum.

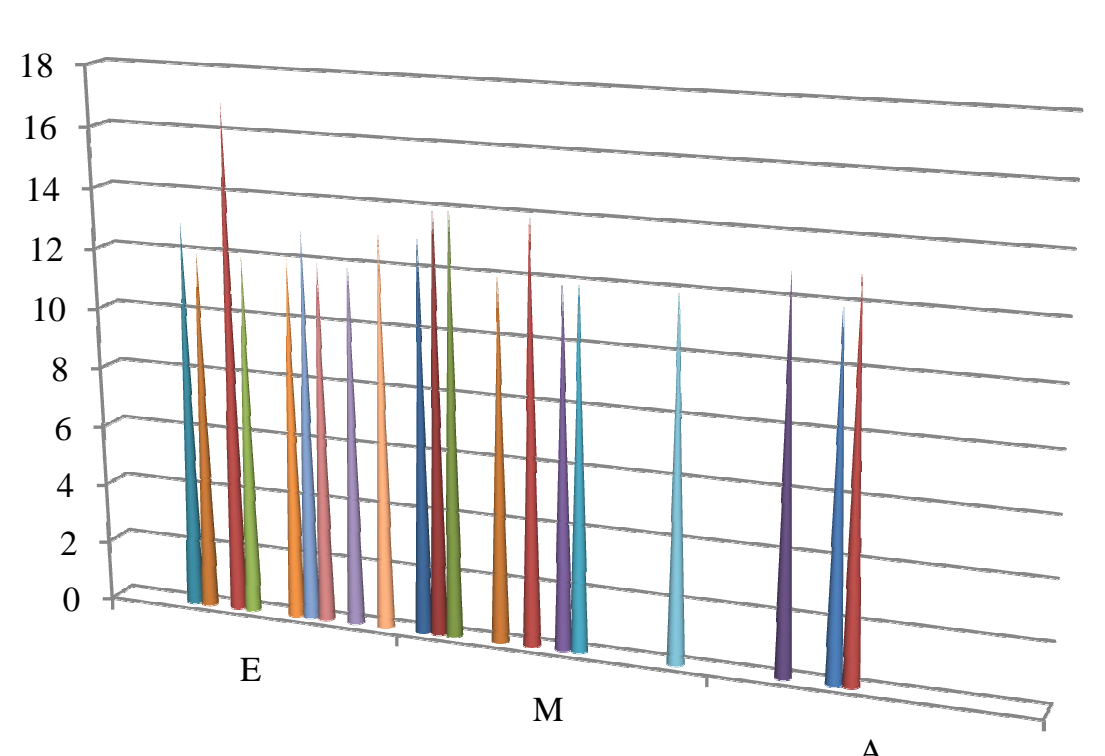

A
- Allium sativam

- Annona reticulata

- Annona squamosa

- Argemone mexicana

- Butea monosperma

- Ceasalpinia bonducella

- Citrus medica

- Corchorus oleterius

- Emblica officinalis

- Euphorbia tirucalli

- Ficus racemosa

- Gymnosporia montana

- Lawsonia inermis

- Solanum nigrum

- Sterculiafoetida

- Tribulus terrestris

Vitex negundo

Zingiber officinale

Fig. 5: Maximum activity of 18 very effective medicinal plants in different solvent extracts against $T$. rubrum.

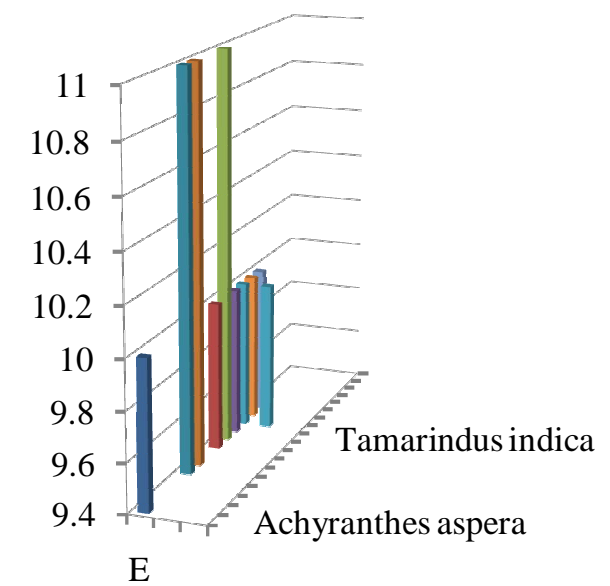

- Achyranthes aspera

- Aloe vera

Bergera koenigii

- Celosia argentea

- Coccinia indica

- Datura metel

- Milletia pinnata

- Senna auriculata

- Senna tora

- Tamarindus indica

- Tectona grandis

Tinospora cordifolia

Thevetia nerrifolia

$\mathrm{E}$

Fig. 6: Maximum activity of 13 effective medicinal plants in different solvent extracts against $T$. rubrum. 


\section{CONCLUSION}

On the basis of the results obtained, we conclude that the 31 effective crude extracts (18 methanolic, 07 ethyalacetate, 04 chloroform and 02 petroleum ether) showed significant antidermatophytic activity against $T$. rubrum. This probably explains the use of these plants by the indigenous people against dermatological infections.

The various phytochemical constituents like saponins, glycosides, tannins, alkaloids and flavonoids present in the crude extract. The purified components may have even more potential with respect to inhibition of microbes. The work carried was a basic approach to find out the antimicrobial activity in $T$. rubrum. Further works on the types of phytoconstituents and purification of individual groups of bioactive components can reveal the exact potential of the plants to inhibit skin pathogenic microbes.

\section{ACKNOWLEDGEMENTS}

The authors are thankful to Gulbarga University for providing lab facilities.

\section{REFERENCES}

Ali-Shtayeh MS, Abu Ghdeib SI. Antifungal activity of plant extracts against dermatophytes. Mycoses, 1999; 42(11-12): 665-72.

Ananthanarayan and Paniker. Textbook of Microbiology, Universities Press, 2009; 8: 604-606.

Baquero F, Gram-positive resistance: Challenges for the development of new antibiotics. Journal of Antimicrobial Chemotherapy, 1997; 39: 1-6.

Bharathi Halu, GM Vidyasagar. A comparative study: differential antimycoses activity of crude leaf extracts of Calotropis spp. Inter J Pharmacy and Pharmaceutical sciences, 2012; 4(3): 705-708.

Chopra, I., Hodgson, J., Metcalf, B., Poste, G., 1996. New approaches to the control of infections caused by antibiotic resistant bacteria. An industry perspective. Journal of the American Medical Association 275, 401-403.

Cowan MM. Plant products as antimicrobial agents. Clin. Microb, 1999; 12:564-583.

David G, Richard CBS, John FP. Medical microbiology. A guide to microbial infections palliogenesis, Immunity, laboratory diagnosis and control, 15th Edi ELST publishers, 1997; 558-564.

Hadacek, F, Greger H. Testing of antifungal natural products: methodologies, comparability of results and assay choise. Phytochem. Anal,2000; 11, 3: 137-147.

Horborne JB. Phytochemical Methods, A Guide to Modern Techniques of Plant Analysis $3^{\text {rd }}$ Eds. Chapman and Hall, 1998; London.

Ibrahim MB. Anti-microbial effects of extract leaf, stem and root bark of Anogeissus leiocarpus on Staphylococcus aureus, Streptococcus pyogenes, Escherichia coli and Proteus vulgaris. J. Pharma Devpt, 1997; 2: 20-30.
Magaldi S, Mata-Essayag S, Hartung de Capriles C, Perez C, Colella MT, Carolina Olaizola, Yudith Ontiveros. Well diffusion for antifungal susceptibility testing. Int. Journal of Infectious Diseases, 2004; 8: $39-45$

Mann A, Banso A,Clifford LC. An antifungal property of crude plant extracts from Anogeissus leiocarpus and Terminalia avicennioides. Tanzania J. Health Res, 2008; 10 (1): 34-38.

Mehmood Z, Ahmad I, Mohammad F, Ahmad D. Indian medicinal plants: A potential source for anticandidal drugs. Pharm Biol, 1999; 37: 237-242.

National Committee for Clinical Laboratory Standards (NCCLS). Approved Standard M2-A6, 5th ed.1997; NCCLS: Wayne, PA.

Ogu GI, Madagwu EC, Eboh OJ, Ezeadila JO. Anti-fungal evaluation of Diodia scandens SW leaf extracts against some dermatophytes in Ukwuani Region of Delta State, Nigeria. Inter. Res. J. of Plant Sci., 2011; 2(10): 311-316.

Ogundipe O, Akinbiyi O, Moody JO. Antibacterial activities of essential ornamental plants. Nigeria J. Natural Products \& Medicine, 1998; 2: 46-47.

Prashanth Kumar V, Neelam Chauhan S, Harish Padh, Rajani M. Search for antibacterial and antifungal agents from selected Indian medicinal plants. Journal of Ethnopharmacology, 2006; 107: 182-188.

Raginee Verma, Satsangi G P and Shrivastava J N. Susceptibility of a weed Calotropis procera (Ait.) against clinical isolates of dermatophytes. J Med Plan Res, 2011; 5(19): 4731-4739.

Santos, DA, Barros MES, Hamdan JS. Establishing a method of inoculum preparation for susceptibility testing of Trichophyton rubrum and Trichophyton mentagrophytes. J. Clin. Microbiol, 2006; 44: 98-101.

Sasidharan VK. Search for antibacterial and antifungal activity some plants of Kerala. Acta Pharmaceut, 1997; 47: 47-51.

Seetharam YN, Kotresh K, Upalaonkar SB. Flora of Gulbarga district. (Gulbarga University, Gulbarga) 2000.

Shinkafi SA, Manga SB. Isolation of Dermatophytes and Screening of selected Medicinal Plants used in the treatment of Dermatophytoses. Int. Res. J. Microbiol, 2011; 2(1):040-048.

Shivakumar singh P, Vidyasagar GM. In-vitro antidermatophytic activity of low polar petroleum ether and inter polar methanolic seed extracts of Sterculia foetida L. Int J Pharm Bio Sci, 2014; 5 (2) : (B) $872-879$.

Sowjanya NC, Manohara Chary C. Effect of plant extracts on the growth of Microsporum gypseum. Journal of Phytology, 2012; 4(2): 41-44.

Sumit Kumar, Shrikara Mallya P, Shalini Shenoy M. Trichophyton rubrum: The Commonest Isolate from Dermatophytosis. Res J Pharm Bio Chem Sci, 2012; 3(1): 101-106.

Taylor RS, Manandhar NP, Towers GHN. Screening of selected medicinal plants of Nepal for antimicrobial activities. Journal of Ethno pharmacology, 1995; $46(3,5): 153-159$.

Weitzman I and Summerbell RC. The dermatophytes. Clin Microbiol Rev, 1995; 8(2): 240-259.

\section{How to cite this article:}

Singh SP and Vidyasagar GM. Antifungal screening of 61 folkloric medicinal plant extracts against dermatophytic fungi Trichophyton rubrum. J App Pharm Sci, 2015; 5 (05): 038-044. 